\section{Transmisión intra-hogar en personas infectadas por SARS-CoV-2 (COVID-19) en Lima, Perú}

\author{
Household transmission of SARS-CoV-2 \\ (COVID-19) in Lima, Peru
}

\author{
Yolanda Angulo-Bazán1 \\ Gilmer Solis-Sánchez 1 \\ Fany Cardenas 1 \\ Ana Jorge 1 \\ Joshi Acosta 1 \\ César Cabezas 1
}

\section{Resumen}

El objetivo fue describir las características de la infección por SARS-CoV-2 entre miembros de hogares, con un caso confirmado primario de COVID-19, en distritos de baja carga de casos en Lima, Perú, en comparación con un distrito de alta carga. Estudio retrospectivo de revisión de base de datos secundaria. Se recolectó información proveniente de una actividad de vigilancia epidemiológica en contactos cercanos (cohabitantes), en 52 hogares de Lima, con un solo miembro con COVID-19. En 10 hogares se realizó una reevaluación. Se evaluaron variables epidemiológicas y clínicas; $y$ su asociación con el resultado a la prueba serológica rápida (presencia de IgG, IgM o ambas). En 40 hogares se encontraron casos secundarios, lo que representa un 49,9\% de identificación en promedio por hogar. Se encontró una tasa de ataque secundaria entre cohabitantes de 53\% (125 casos), siendo sintomáticos un 77,6\% de casos (razón sintomáticos/asintomáticos: 3,5). La presencia de fiebre y/o escalofríos se encontró en el $40 \%$ de las personas con resultado positivo, seguido del dolor de garganta en el 39,2\%. La ageusia y anosmia estuvieron presentes en el 22,4\% y 20,8\% de los casos, respectivamente. Al tener un caso primario de COVID-19 en el hogar, la tasa de ataque secundaria de esta infección es de 53\%; sin embargo, en una proporción importante de hogares evaluados no hubo un caso positivo, más allá del caso primario. Las características epidemiológicas y clínicas encontradas en este caso estuvieron acorde a lo ya reportado en otras series internacionales.

COVID-19; Trazado de Contacto; Servicios de Vigilancia Epidemiológica
Correspondencia

Y. Angulo-Bazán

Capac Yupanqui 1400. Jesús María, Lima 15073, Perú. yangulobazan@gmail.com

1 Instituto Nacional de Salud, Lima, Perú. 


\section{Introducción}

El SARS-CoV-2 es un virus RNA, perteneciente a la familia de los Orthocoronavirinae, a la cual también pertenecen otros agentes causantes de pandemias como el síndrome respiratorio de Oriente Medio (MERS-CoV) o síndrome respiratorio agudo grave (SARS-CoV) 1. En Perú, el primer caso de COVID-19 fue identificado el 6 de marzo del 2020, mientras que las dos primeras muertes por esta enfermedad se dieron trece días después 2,3 . Tres meses después, el país supera los 260.000 casos y reporta más de 8.700 fallecidos (Ministerio de Salud. Sala situacional COVID-19 Perú. https:// covid19.minsa.gob.pe/, accedido el 08/May/2020).

Una de las características más importantes del COVID-19 es su dinámica de transmisibilidad; debido a sus mecanismos altamente eficaces de transmisión. Este agente infeccioso se propaga usualmente por vía respiratoria o por contacto con secreciones; por ende, la transmisión humano-humano se ha convertido en la principal vía de diseminación a ser manejada en esta pandemia ${ }^{4}$. Estudios previos han determinado que el SARS-CoV-2 posee números básicos de reproducción (R0) promedio de 2,2 , pero que pueden oscilar entre 1,4 a 6,5; sin embargo, estas estimaciones pueden variar de acuerdo al contexto de estudio 5,6 .

Los contactos cercanos a los casos, como miembros de la familia, parientes o amigos, son aquellos que se encuentran en mayor riesgo de contraer la infección y, por consiguiente, pueden ser fuentes de contagio para otras personas que se encuentren en contacto con ellos. Esta cadena de contagio se ve reforzada por el hecho de que un porcentaje de la población de infectados puede actuar como portadores asintomáticos de la enfermedad, lo que dificulta su identificación por los sistemas de salud 7.

En ese aspecto, una forma eficaz de romper la cadena de transmisión del virus SARS-CoV-2 es a través de la vigilancia epidemiológica y seguimiento de todas las personas que estuvieron en contacto cercano con un caso confirmado 8,9. Este proceso se denomina "estudio de contactos" o "contact tracing". Algunas experiencias previas han utilizado estas estrategias para evaluar la dinámica de transmisión entre cohabitantes de COVID-19. Un estudio realizado en China menciona que existió una correlación entre los casos confirmados en otras comunas de la provincia de Hubei, y la cantidad de migrantes de Wuhan, que usualmente venían a hacer visitas familiares 10. Así mismo, Liu et al. 11 evidenciaron que los eventos de reuniones familiares se convirtieron en fuentes importantes de contagio en algunas provincias de China, por lo que recomiendan que las intervenciones de salud pública deben considerar medidas específicas para la reducción del contacto en cohabitantes.

Adicionalmente, otras investigaciones han encontrado que las tasas de ataque secundario se incrementan entre 7-10 veces, cuando se estudia solo a las personas que cohabitan la vivienda con el caso primario, en comparación con la tasa calculada cuando se incluyen a todos los que estuvieron en contacto con este 12,13. Sin embargo, la evidencia es aún divergente entre regiones y países en los que se realizan los estudios de contactos.

En América Latina, la debilidad de los sistemas de salud y la falta de recursos económicos se agregan a la dificultad al seguimiento de casos y contactos en esta enfermedad, esto ha demostrado ser un factor gravitante en la progresión de esta pandemia 14. Benítez et al. 15, en un análisis reciente en cinco países latinoamericanos, sugieren que la ejecución de un seguimiento estricto de contactos, como en Chile, se relaciona con disminuciones sostenidas de casos de COVID-19. Adicionalmente, el estudio de contactos se ha venido implementando de manera tardía en la región, siendo aún incompleto en los países con tasas altas de mortalidad como Brasil, Ecuador o Perú 16.

En nuestro país, el centralismo se adiciona como un factor adicional; es así que, en la capital, Lima Metropolitana, se concentra aproximadamente el 60\% de los casos a nivel nacional. Adicionalmente, dentro de ella, se han identificado distritos con alta y baja proporción de casos, los que han ido variando en el tiempo (Ministerio de Salud. Sala situacional COVID-19 Perú. https://covid19.minsa. gob.pe/, accedido el 08/May/2020).

Si bien se ha encontrado un estudio previo que analiza de manera preliminar la dinámica de la transmisión de SARS-CoV-2 en Lima 17, no se han encontrado análisis que evalúen la información de actividades que hayan involucrado el seguimiento de conglomerados de contactos estrechos, como lo son las personas que habitan el mismo hogar, considerando la carga por distritos de residencia. Por ello, el objetivo del presente estudio es describir las características de la infección por SARS-CoV-2 
entre miembros de hogares con un caso confirmado primario de COVID-19 en distritos de baja carga de casos en Lima Metropolitana, en comparación con un distrito de alta carga.

\section{Materiales y métodos}

\section{Diseño y tipo de estudio}

El presente estudio se plantea dentro del enfoque cuantitativo; siendo de tipo observacional y retrospectivo.

\section{Población y muestra}

La población de estudio se definió como la totalidad de las fichas de reporte de resultados de prueba serológica inmunocromatográfica de flujo lateral para COVID-19. Como criterio de inclusión, se seleccionaron contactos con fichas epidemiológicas completas con resultados de $\operatorname{IgG} / \mathrm{IgM}$, realizadas por personal del Instituto Nacional de Salud del Perú (INS), incluidos en la vigilancia epidemiológica de hogares. Se excluyeron a aquellas fichas que no se hayan encontrado en el proceso de búsqueda, o que pertenezcan a personas que no cohabitan en el domicilio del caso primario. Por ende, este estudio se considera de tipo censal.

\section{Vigilancia epidemiológica}

En el marco del control y vigilancia de la pandemia, el INS realizó una actividad de vigilancia epidemiológica de hogares con un solo caso primario COVID-19 (identificado por RT-PCR), entre el 23 de abril y el 2 de mayo del 2020. Esta evaluación se realizó, en promedio, a 13,6 $\pm 3,7$ días posteriores a la realización de la prueba diagnóstica.

Con el fin de evitar la inclusión de casos de infección por contagio en ambientes fuera del hogar, esta actividad se realizó en los distritos con menor carga en cada una de las cuatro jurisdicciones descentralizadas de salud de Lima, denominadas Direcciones de Redes Integradas de Salud (DIRIS), hasta alcanzar la vigilancia de 10 hogares por DIRIS. Para ello, se obtuvieron los resultados de pruebas moleculares (RT-PCR), registradas desde el 9 de abril en el sistema NetLab v.2.0 del Ministerio de Salud (https://netlabv2.ins.gob.pe/Login).

Los resultados identificados se agruparon por distrito de residencia; y fueron ordenados por carga de casos dentro de cada una de sus DIRIS, tras lo cual se seleccionaron, de forma no probabilística intencional, a 10 hogares de los distritos con menor carga de casos en cada DIRIS. En la DIRIS correspondiente a la zona céntrica de Lima (de mayor densidad poblacional), se consideraron dos hogares adicionales; además, se evalúo el distrito de Lima Metropolitana con mayor proporción de casos, al momento del inicio de la vigilancia. En total, 52 hogares fueron incluidos en este estudio.

Posteriormente, como parte de la vigilancia, se reevaluaron los doce hogares ubicados en la DIRIS correspondiente a la zona céntrica de Lima, en promedio a los 33,6 \pm 2,7 días después de la primera evaluación.

La prueba serológica utilizada fue Coretests COVID-19 IgM/IgG Ab Test (Core Technology Co., Pekín, China), una prueba inmunocromatográfica de flujo lateral que detecta cualitativamente la presencia de anticuerpos contra SARS-CoV-2, con una sensibilidad y especificidad para $\operatorname{IgM} / \operatorname{IgG}$ de $97,6 \%$ y $100 \%$, reportada por el fabricante; dichos valores fueron verificados por el INS, a través de evaluaciones a nivel de laboratorio, reportándose un $96,4 \%$ de sensibilidad y $96 \%$ de especificidad, tanto para IgG como para $\operatorname{IgM} 18$. 


\section{Variables}

El estudio contempla una variable principal denominada como infección por SARS-CoV-2, y definida como la presencia de anticuerpos (IgM, IgG o ambos) en personas en las que previamente no se había tenido alguna prueba con resultado positivo (RT-PCR o prueba serológica). Los casos positivos fueron clasificados a su vez, de acuerdo a la presencia/ausencia de síntomas.

Así mismo, se recolectó información sobre la cantidad de miembros en el hogar, tiempo de evaluación, definido como el tiempo en días entre la emisión del resultado del caso índice y la primera evaluación; y el tiempo de enfermedad, caracterizado como el tiempo en días (referido por el paciente) desde el inicio de síntomas hasta el día de la evaluación.

También se describieron características sociodemográficas de los miembros del hogar (edad, sexo, presencia de profesionales de la salud), y características clínicas (presencia de síntomas y condiciones de riesgo). Al respecto, se consideraron como síntomas: presencia de tos, dolor de garganta, congestión nasal, fiebre, malestar general, dificultad respiratoria, diarrea, náuseas/vómito, cefalea, irritabilidad/confusión, dolor en general, entre otros; mientras que se incluyeron como condiciones de riesgo: edad mayor o igual a 60 años, hipertensión arterial, enfermedad cardiovascular, diabetes tipo 2, obesidad, asma, enfermedad pulmonar crónica, insuficiencia renal crónica (IRC), enfermedad o tratamiento inmunosupresor, cáncer, embarazo o puerperio, ser profesional de la salud u otras que el personal de salud consideró conveniente registrar 19.

\section{Análisis estadístico}

El análisis estadístico descriptivo de los datos fue llevado a cabo mediante la determinación de la frecuencia, porcentaje, media y desviación estándar de los datos recolectados. Dicha evaluación se realizó de forma diferenciada, expresando medidas simples para la información de los sujetos de manera general; mientras que, para identificar los valores de las personas dentro de cada hogar se utilizaron medidas promedio, considerando la variabilidad que existe en cada hogar, según la densidad de integrantes que posee. Se repitieron los análisis para las personas y hogares reevaluados, a fin de identificar los cambios ocurridos en el tiempo. El software estadístico utilizado fue Stata versión 16.0 (https://www.stata.com).

\section{Aspectos éticos}

Debido a que el presente estudio utilizó fuentes secundarias de información, en el marco de una actividad de vigilancia epidemiológica, no se requirió de un consentimiento informado. Se preservó la confidencialidad de los datos personales de los participantes mediante el uso de bases de datos anónimas, y no se recolectó información que permitiera la identificación de las personas incluidas. El estudio cuenta con la aprobación por el Comité Institucional de Ética en Investigación del INS (RD no 256-2020-OGITT/INS).

\section{Resultados}

Se evaluaron registros de 326 personas, en los que se encontró que 54,7\% ( $n=129$ ) eran mujeres, con un promedio de edad de 36,2 $\pm 20,1$. El tiempo promedio desde la detección del caso primario y la evaluación de los contactos, fue de 13,6 \pm 3,7 días. El 37,3\% presentaba alguna condición de riesgo ( $\mathrm{n}=88$ ), siendo la más frecuente el pertenecer al grupo etario riesgo de 60 años a más ( $\mathrm{n}=35,39,8 \%)$, seguido de hipertensión arterial $(n=20,22,7 \%)$ y asma bronquial $(n=14,15,9 \%)$. En lo referente a los aspectos semiológicos de las personas, 68,6\% presentaron algún signo y/o síntoma; destacándose la presencia de dolor de garganta (49,4\%), mientras que la fiebre y/o escalofríos, así como la tos se presentaron en el $41,4 \%$ de las personas.

De la totalidad de sujetos, el 53\% fue identificado como caso secundario al obtener resultado positivo mediante prueba inmunocromatográfica de flujo lateral, encontrándose 15 personas solo con reacción positiva a IgM, y 110 con reacción tanto para IgM e IgG, no se encontraron pacientes 
que exhibieran solamente reacción positiva a IgG. Entre los casos secundarios, se observó que el $77,6 \%$ eran sintomáticos, la razón de casos secundarios sintomáticos sobre los asintomáticos fue de 3,5 (Tabla 1).

Se encontraron edades similares entre las personas clasificadas por resultados (positivo/negativo) y por síntomas (sintomáticos/asintomáticos). El 40,2\% de los casos sintomáticos y el 32,1\% de los asintomáticos tenían alguna condición de riesgo, siendo la característica más frecuentemente hallada, el pertenecer al grupo etario mayor de 60 años. Los signos y síntomas más frecuentemente encontrados, en los casos positivos, fueron fiebre y/o escalofríos (40\%), dolor de garganta (39,2\%), tos (35,2\%), cefalea $(30,4 \%)$ y malestar general (28\%). La ageusia y anosmia, estuvieron presentes en el 22,4\% y 20,8\% de los casos, respectivamente. El tipo de inmunoglobulina detectada se presentó semejantemente entre casos secundarios sintomáticos y asintomáticos (Tabla 2).

Las 236 personas evaluadas pertenecieron a 52 hogares, encontrándose una densidad de 4,5 \pm 2,5 integrantes por cada hogar; considerando la variabilidad en la cantidad de integrantes por hogar, se encontró que el 54,1\% de los miembros fueron mujeres, el 34,7\% de los integrantes por hogar tuvo

\section{Tabla 1}

Características de las personas evaluadas en general. Lima, Perú.

\begin{tabular}{|c|c|}
\hline & $\begin{array}{l}\text { Personas en general } \\
\qquad \begin{array}{c}(n=236) \\
n(\%)\end{array}\end{array}$ \\
\hline Edad (años) [media $\pm \mathrm{DE}]$ & $36,2 \pm 20,1$ \\
\hline \multicolumn{2}{|l|}{ Sexo } \\
\hline Masculino & $107(45,3)$ \\
\hline Femenino & $129(54,7)$ \\
\hline \multicolumn{2}{|c|}{ Distrito de residencia según carga de casos } \\
\hline Baja carga & $185(78,4)$ \\
\hline Alta carga & $51(21,6)$ \\
\hline \multicolumn{2}{|l|}{ Presencia de condición de riesgo } \\
\hline No presenta & $148(62,7)$ \\
\hline Sí presenta & $88(37,3)$ \\
\hline \multicolumn{2}{|l|}{ Condición de riesgo identificada * } \\
\hline Diabetes & $13(14,8)$ \\
\hline Hipertensión arterial crónica & $20(22,7)$ \\
\hline Asma bronquial & $14(15,9)$ \\
\hline Insuficiencia renal & $1(1,14)$ \\
\hline Cardiopatía & $7(8,0)$ \\
\hline Obesidad & $9(10,2)$ \\
\hline Antecedente de neumonía & $2(2,3)$ \\
\hline Fibromialgia & $1(1,1)$ \\
\hline Anemia & $3(3,4)$ \\
\hline Hipotiroidismo & $7(8,0)$ \\
\hline Tuberculosis & $3(3,4)$ \\
\hline Cáncer & $4(4,6)$ \\
\hline Enfermedad autoinmune & $1(1,1)$ \\
\hline Profesional de la salud & $9(10,2)$ \\
\hline Grupo etario de riesgo & $35(39,8)$ \\
\hline \multicolumn{2}{|l|}{ Presencia de signos y/o síntomas } \\
\hline No presenta & $74(31,4)$ \\
\hline Sí presenta & $162(68,6)$ \\
\hline
\end{tabular}

(continúa) 


\begin{tabular}{|c|c|}
\hline & $\begin{array}{l}\text { Personas en general } \\
\qquad(n=236) \\
n(\%)\end{array}$ \\
\hline Tiempo de enfermedad (días) ${ }^{* \star}[$ media $\pm \mathrm{DE}]$ & $11,8 \pm 7,5$ \\
\hline \multicolumn{2}{|l|}{ Signos y síntomas identificados ** } \\
\hline Tos & $67(41,4)$ \\
\hline Dolor de garganta & $80(49,4)$ \\
\hline Congestión nasal & $35(21,6)$ \\
\hline Dificultad respiratoria & $22(13,6)$ \\
\hline Fiebre/Escalofrío & $67(41,4)$ \\
\hline Malestar general & $56(34,6)$ \\
\hline Diarrea & $27(16,7)$ \\
\hline Náuseas/Vómitos & $7(4,3)$ \\
\hline Cefalea & $62(38,3)$ \\
\hline Irritabilidad/Confusión & $4(2,5)$ \\
\hline Dolor & $1(0,6)$ \\
\hline Anosmia & $28(17,3)$ \\
\hline Ageusia & $31(19,1)$ \\
\hline \multicolumn{2}{|l|}{ Resultado de la prueba } \\
\hline Negativo & $111(47,0)$ \\
\hline Positivo & $125(53,0)$ \\
\hline Razón de positivos y negativos & 1,1 \\
\hline \multicolumn{2}{|l|}{ Inmunoglobulina detectada } \\
\hline Solo IgM & $15(12,0)$ \\
\hline IgM/IgG & $110(88,0)$ \\
\hline Solo IgG & $0(0,0)$ \\
\hline \multicolumn{2}{|l|}{ Tipo de casos positivos } \\
\hline Asintomático & $28(22,4)$ \\
\hline Sintomático & $97(77,6)$ \\
\hline Razón de positivos sintomáticos y asintomáticos & 3,5 \\
\hline
\end{tabular}

DE: desviación estándar.

* Los valores fueron calculados considerando solo a quienes sí presentaban alguna condición de riesgo;

** Los valores fueron calculados considerando solo a quienes sí presentaban algún signo y/o síntoma.

alguna condición de riesgo, y el 68,1\% presentó algún signo y/o síntoma. En promedio, el 49,9\% de los integrantes de cada hogar fue identificado como caso secundario para COVID-19; de los 40 hogares que presentaron casos secundarios, en $9(22,5 \%)$ la totalidad de sus integrantes tuvieron resultado positivo. Se encontró que, en promedio, el 39,4\% de los integrantes de casa fueron casos secundarios sintomáticos, la razón de encontrar casos secundarios sintomáticos fue de 3,8, con respecto a los asintomáticos (Tabla 3).

Al evaluar las características de los hogares según la positividad de sus integrantes, se encontró que en aquellos donde la totalidad de sus miembros fueron positivos el $66,7 \%$ eran mujeres, mientras que en donde todos eran negativos esta cifra fue de $55 \%$, y de $50 \%$, donde se tuvieron tanto integrantes positivos como negativos. Con respecto a condiciones de riesgo, se encontró mayor frecuencia conforme los hogares tenían más integrantes positivos (Tabla 4).

En los datos de reevaluación se consideraron a 40 personas distribuidas en 12 hogares, con un promedio de edad de las personas incluidas de 34,2 $\pm 17,2$ años. Las personas fueron reevaluadas a los $33,6 \pm 2,7$ días después de la primera evaluación. Se encontró una media de 66,8\% integrantes mujeres por hogar y $39,6 \%$ personas con condición de riesgo por hogar. 
Características de las personas con resultado positivo y negativo, según presencia de síntomas.

\begin{tabular}{|c|c|c|c|c|c|c|}
\hline & \multicolumn{3}{|c|}{ Persona negativa } & \multicolumn{3}{|c|}{ Persona positiva } \\
\hline & $\begin{array}{l}\text { Sin síntomas } \\
\qquad \begin{array}{c}(\mathrm{n}=46) \\
\mathrm{n}(\%)\end{array}\end{array}$ & $\begin{array}{l}\text { Con síntomas } \\
\qquad \begin{array}{c}(n=65) \\
n(\%)\end{array}\end{array}$ & $\begin{array}{c}\text { Total } \\
(n=111) \\
n(\%)\end{array}$ & $\begin{array}{l}\text { Sin síntomas } \\
\qquad \begin{array}{c}(\mathrm{n}=\mathbf{2 8}) \\
\mathrm{n}(\%)\end{array}\end{array}$ & $\begin{array}{l}\text { Con síntomas } \\
\qquad \begin{array}{c}(\mathrm{n}=97) \\
\mathrm{n}(\%)\end{array}\end{array}$ & $\begin{array}{c}\text { Total } \\
(n=125) \\
n(\%)\end{array}$ \\
\hline Edad (años) [media \pm DE] & $37,7 \pm 18,9$ & $33,1 \pm 19,1$ & $35,0 \pm 19,1$ & $39,5 \pm 21,2$ & $36,5 \pm 20,9$ & $37,2 \pm 20,9$ \\
\hline \multicolumn{7}{|l|}{ Sexo } \\
\hline Masculino & $22(47,8)$ & $31(47,7)$ & $53(47,8)$ & $11(39,3)$ & $43(44,3)$ & $54(43,2)$ \\
\hline Femenino & $24(52,2)$ & $34(52,3)$ & $58(52,2)$ & $17(60,7)$ & $54(55,7)$ & $71(56,8)$ \\
\hline \multicolumn{7}{|l|}{ Presencia de signos y/o síntomas } \\
\hline No presenta & $24(52,2)$ & $47(77,3)$ & $71(64,0)$ & $19(67,9)$ & $58(59,8)$ & $77(61,6)$ \\
\hline Sí presenta & $22(47,8)$ & $18(27,7)$ & $40(36,0)$ & $9(32,1)$ & $39(40,2)$ & $48(38,4)$ \\
\hline \multicolumn{7}{|l|}{ Condición de riesgo } \\
\hline Anemia & $1(2,2)$ & $0(0,0)$ & $1(0,9)$ & $0(0,0)$ & $2(2,1)$ & $2(1,6)$ \\
\hline Antecedente de neumonía & $0(0,0)$ & $1(1,5)$ & $1(0,9)$ & $1(3,6)$ & $0(0,0)$ & $1(0,8)$ \\
\hline Asma bronquial & $4(8,7)$ & $2(3,1)$ & $6(5,4)$ & $1(3,6)$ & $7(7,2)$ & $8(6,4)$ \\
\hline Cáncer & $1(2,2)$ & $1(1,5)$ & $2(1,8)$ & $1(3,6)$ & $1(1,0)$ & $2(1,6)$ \\
\hline Cardiopatía & $1(2,2)$ & $2(3,1)$ & $3(2,7)$ & $1(3,6)$ & $3(3,1)$ & $4(3,2)$ \\
\hline Diabetes & $5(10,9)$ & $1(1,5)$ & $6(5,4)$ & $1(3,6)$ & $6(6,2)$ & $7(5,6)$ \\
\hline Enfermedad autoinmune & $0(0,0)$ & $0(0,0)$ & $0(0,0)$ & $0(0,0)$ & $1(1,0)$ & $1(0,8)$ \\
\hline Fibromialgia & $0(0,0)$ & $0(0,0)$ & $0(0,0)$ & $0(0,0)$ & $1(1,0)$ & $1(0,8)$ \\
\hline Grupo etario de riesgo & $7(15,2)$ & $5(7,7)$ & $12(10,8)$ & $6(21,4)$ & $17(17,5)$ & $23(18,4)$ \\
\hline Hipertensión arterial crónica & $6(13,0)$ & $5(7,7)$ & $11(9,9)$ & $3(10,7)$ & $6(6,2)$ & $9(7,2)$ \\
\hline Hipotiroidismo & $3(6,5)$ & $2(3,1)$ & $5(4,5)$ & $0(0,0)$ & $2(2,1)$ & $2(1,6)$ \\
\hline Insuficiencia renal & $1(2,2)$ & $0(0,0)$ & $1(0,9)$ & $0(0,0)$ & $0(0,0)$ & $0(0,0)$ \\
\hline Obesidad & $3(6,5)$ & $2(3,1)$ & $5(4,5)$ & $1(3,6)$ & $3(3,1)$ & $4(3,2)$ \\
\hline Profesional de la salud & $1(2,2)$ & $2(3,1)$ & $3(2,7)$ & $1(3,6)$ & $5(5,2)$ & $6(4,8)$ \\
\hline Tuberculosis & $0(0,0)$ & $2(3,1)$ & $2(1,8)$ & $0(0,0)$ & $1(1,0)$ & $1(0,8)$ \\
\hline \multicolumn{7}{|l|}{ Inmunoglobulina detectada } \\
\hline Solo IgM & - & - & - & $3(10,7)$ & $12(12,4)$ & $15(12,0)$ \\
\hline IgM/lgG & - & - & - & $25(89,3)$ & $85(87,6)$ & $110(88,0)$ \\
\hline Solo IgG & - & - & - & $0(0,0)$ & $0(0,0)$ & $0(0,0)$ \\
\hline
\end{tabular}

DE: desviación estándar.

En la primera visita, se calculó un promedio de 1,9 \pm 1,4 de habitantes por hogar que tuvieron algún signo y/o síntoma (59,2\%); mientras que, para la reevaluación, esta cantidad fue de 0,9 \pm 0,5 (41,6\%). En la primera evaluación, se encontró 1,8 \pm 1,5 casos positivos por hogar (57\%); mientras que para la reevaluación este promedio fue 2,0 \pm 1,5 (65,6\%). Con ello se pudo identificar que la razón de casos positivos en los integrantes por hogar pasó de 1,33 a 1,91. Todos los casos IgM+IgG positivos en la primera evaluación presentaron IgM+IgG positiva en la reevaluación.

El único caso reactivo solo a IgM en la primera evaluación presentó también IgG para la segunda evaluación; adicionalmente, se identificaron tres casos que inicialmente eran negativos y en la segunda visita exhibieron reacción positiva para IgM e IgG. El promedio de casos positivos sintomáticos por hogar, en la primera visita, fue de 1,3 $\pm 1,4$ (44,6\%); en cambio, para la segunda evaluación se encontró una media de $0,8 \pm 0,4(37,4 \%)$. La razón de positivos sintomáticos sobre los asintomáticos cambió de 3,60 a 1,33 (Tabla 5). 
Tabla 3

Características de la composición de los hogares (personas por hogar).

\section{Características de los integrantes por hogar}

Integrantes por hogar *

Edad (años) *

Sexo (\%)

Masculino

Femenino

Personas por hogar con presencia de comorbilidades (\%)

No presenta

Sí presenta

Personas por hogar con presencia de signos y/o síntomas (\%)

No presenta

Sí presenta

Tiempo de enfermedad (días) *

Resultado de prueba serológica rápida de personas por hogar (\%)

Negativo

Positivo

Personas con IgM reactivo por hogar (\%)

Negativo

Positivo

Personas con IgG reactivo por hogar (\%)

Negativo

Positivo

Hogares según casos positivos [n (\%)]

Hogares donde todas las personas fueron negativas

Hogares donde al menos 1 persona fue positiva

Hogares con personas negativas y positivas

Hogares donde todas las personas fueron positivas

Hogares con casos positivos según presencia de signos y síntomas [n (\%)]

Hogares donde al menos 1 persona fue positiva asintomática

Hogares donde al menos 1 persona fue positiva sintomática

Razón de hogares con al menos 1 persona positiva sintomática y asintomática

Distribución de personas según sus resultados por hogar (\%)

Personas negativas por hogar

Personas positivas asintomáticas por hogar

Personas positivas sintomáticas por hogar

Razón de personas positivas sintomáticas y asintomáticas por hogar
Hogares en general

$(n=52)$

$4,5 \pm 2,5$

$36,2 \pm 20,1$

45,9

54,1

65,3

34,7

31,9

68,1

$12,1 \pm 7,3$

50,1

49,9

50,1

49,9

57,1

$12(23,1)$

$40(76,9)$

$31(77,5)$

$9(22,5)$

$11(76,9)$

$35(23,1)$

3,2

50,1

10,5

39,4

3,8

Nota: los valores porcentuales corresponden al porcentaje promedio de la característica de las personas por hogar. * Los valores corresponden al promedio de la característica de las personas por hogar con presencia de algún signo y/o síntoma. 
Características de la composición de los hogares (personas por hogar) según resultado de sus integrantes.

\begin{tabular}{|c|c|c|c|}
\hline & $\begin{array}{l}\text { Hogares con totalidad de } \\
\text { integrantes negativos } \\
\qquad(n=12)\end{array}$ & $\begin{array}{l}\text { Hogares con integrantes } \\
\text { positivos y negativos } \\
(n=31)\end{array}$ & $\begin{array}{l}\text { Hogares con totalidad de } \\
\text { integrantes positivos } \\
\qquad(n=9)\end{array}$ \\
\hline Número de personas & 41 & 162 & 33 \\
\hline Integrantes por hogar * & $3,4 \pm 2,0$ & $5,2 \pm 2,3$ & $3,7 \pm 3,1$ \\
\hline Edad (años) * & $36,4 \pm 18,9$ & $36,8 \pm 20,3$ & $33,0 \pm 20,7$ \\
\hline \multicolumn{4}{|l|}{ Sexo (\%) } \\
\hline Masculino & 45,0 & 50,0 & 33,3 \\
\hline Femenino & 55,0 & 50,0 & 66,7 \\
\hline \multicolumn{4}{|l|}{ de signos y/o síntomas (\%) } \\
\hline No presenta & 52,0 & 28,6 & 16,4 \\
\hline Sí presenta & 48,0 & 71,4 & 83,6 \\
\hline Tiempo de enfermedad (días) * & $25,7 \pm 4,9$ & $25,0 \pm 8,2$ & $21,8 \pm 6,5$ \\
\hline \multicolumn{4}{|l|}{$\begin{array}{l}\text { Personas por hogar con condición } \\
\text { de riesgo (\%) }\end{array}$} \\
\hline No presenta & 70,5 & 64,1 & 62,7 \\
\hline Sí presenta & 29,5 & 35,9 & 37,3 \\
\hline $\begin{array}{l}\text { Hogares con algún integrante que } \\
\text { sea profesional de la salud }\end{array}$ & 4 & 4 & 2 \\
\hline
\end{tabular}

Nota: los valores porcentuales corresponden al porcentaje promedio de la característica de las personas por hogar.

* Los valores corresponden al promedio de la característica de las personas por hogar con presencia de algún signo y/o síntoma.

\section{Discusión}

Esta investigación encontró una tasa de ataque secundaria entre cohabitantes de 53\%, lo que es más alto a lo hallado en otras investigaciones que evaluaron la transmisión de SARS-CoV-2 en conglomerados similares. El estudio que obtuvo resultados más similares a los mostrados fue el del Wu et al. 20, en el que se evaluaron a 148 contactos cercanos, todos cohabitantes de un caso primario, en China. En esta publicación, se encontró una tasa de ataque secundario de 32,4\% (IC95\%: 22,4-44,4).

En otros antecedentes, encontrados en países como China, Estados Unidos o Corea del Sur; las tasas de ataque secundario en cohabitantes oscilaron entre 4,6\% a 17\%. Sin embargo, se debe considerar que estas estimaciones se ven afectadas por el tamaño de muestra obtenido, que fue desde un mínimo de 151 y un máximo de 2.370 cohabitantes de casos confirmados 12,21,22,23. Esta divergencia puede ser explicada por las diferencias sociales y culturales entre los países en los que estos estudios han sido desarrollados, así como las medidas de aislamiento social y cuarentena aplicadas por el estado. Se debe mencionar que no se han encontrado antecedentes de estudios similares en América Latina, por lo que no se conoce la verdadera magnitud de la influencia de estos factores en la progresión del contagio de COVID-19 en el entorno del hogar.

Otra explicación a estos resultados es el tiempo trascurrido entre la detección del caso primario y el caso secundario, que en esta investigación fue en promedio 13 días. Guan et al. 24 encontraron, en un seguimiento de contactos que residían en la misma vivienda, que 13 días después de la detección del primer caso, más de la mitad de los casos secundarios ya habían sido identificados. De forma similar, Qian et al. 25 evidenciaron un 88,8\% de detección de casos secundarios en el mismo hogar, en un seguimiento realizado en China.

Al observar las características epidemiológicas de los casos secundarios encontrados, se evidencia un promedio de edad de 36,1 \pm 20,1 años, con un 54,7\% de casos de sexo femenino. Esta distribución es coherente con lo encontrado por la revisión sistemática de Lovato \& De Phillips 26, la que se encon- 
Variación de las características de las personas en general y personas por hogar en la primera evaluación y reevaluación.

\begin{tabular}{|c|c|c|c|c|}
\hline & \multicolumn{2}{|c|}{ Primera evaluación } & \multicolumn{2}{|c|}{ Reevaluación } \\
\hline & $\begin{array}{l}\text { Evaluación de } \\
\text { personas } \\
\quad(n=40) \\
\text { Frecuencia (\%) }\end{array}$ & $\begin{array}{l}\text { Evaluación de personas } \\
\text { por hogar } \\
(n=12) \\
\text { Media } \pm \text { DE }(\%)\end{array}$ & $\begin{array}{l}\text { Evaluación de } \\
\text { personas } \\
\quad(n=40) \\
\text { Frecuencia (\%) }\end{array}$ & $\begin{array}{l}\text { Evaluación de personas } \\
\text { por hogar } \\
(n=12) \\
\text { Media } \pm \text { DE (\%) }\end{array}$ \\
\hline Edad (años) & $34,2 \pm 17,2$ & - & - & - \\
\hline \multicolumn{5}{|l|}{ Sexo } \\
\hline Masculino & $14(35,0)$ & $1,2 \pm 0,9(33,2)$ & - & - \\
\hline Femenino & $26(65,0)$ & $2,2 \pm 1,6(66,8)$ & - & - \\
\hline \multicolumn{5}{|l|}{$\begin{array}{l}\text { Presencia de condición de } \\
\text { riesgo }\end{array}$} \\
\hline No presenta & $24(60,0)$ & $2,0 \pm 1,5(60,4)$ & - & - \\
\hline Sí presenta & $16(40,0)$ & $1,3 \pm 1,4(39,6)$ & - & - \\
\hline \multicolumn{5}{|l|}{$\begin{array}{l}\text { Presencia de signos y/o } \\
\text { síntomas }\end{array}$} \\
\hline No presenta & $17(42,5)$ & $1,4 \pm 1,2(40,8)$ & $29(72,5)$ & $2,4 \pm 2,1(58,4)$ \\
\hline Sí presenta & $23(57,5)$ & $1,9 \pm 1,4(59,2)$ & $11(27,5)$ & $0,9 \pm 0,5(41,6)$ \\
\hline \multicolumn{5}{|l|}{ Resultado de la prueba } \\
\hline Negativo & $19(47,5)$ & $1,6 \pm 1,7(43,0)$ & $16(40,0)$ & $1,3 \pm 1,7(34,4)$ \\
\hline Positivo & $21(52,5)$ & $1,8 \pm 1,5(57,0)$ & $24(60,0)$ & $2,0 \pm 1,5(65,6)$ \\
\hline $\begin{array}{l}\text { Razón de personas positivas } \\
\text { y negativas }\end{array}$ & 1,1 & 1,3 & 1,5 & 1,9 \\
\hline \multicolumn{5}{|l|}{ Inmunoglobulina detectada } \\
\hline Solo IgM & $1(2,5)$ & $0,1 \pm 0,3(8,3)$ & $0(0,0)$ & $0,0 \pm 0,0(0,0)$ \\
\hline $\operatorname{lgM} / \operatorname{lgG}$ & $20(50,0)$ & $1,7 \pm 1,6(48,7)$ & $24(60,0)$ & $2,0 \pm 1,5(65,6)$ \\
\hline Solo IgG & $0(0,0)$ & $0,0 \pm 0,0(0,0)$ & $0(0,0)$ & $0,0 \pm 0,0(0,0)$ \\
\hline \multicolumn{5}{|l|}{ Tipo de casos secundarios } \\
\hline Asintomático & $5(12,5)$ & $0,4 \pm 0,7(12,4)$ & $14(35,0)$ & $1,2 \pm 1,4(28,2)$ \\
\hline Sintomático & $16(40,0)$ & $1,3 \pm 1,4(44,6)$ & $10(25,0)$ & $0,8 \pm 0,4(37,4)$ \\
\hline $\begin{array}{l}\text { Razón de personas positivas } \\
\text { sintomáticas y asintomáticas }\end{array}$ & 3,2 & 3,6 & 0,7 & 1,3 \\
\hline
\end{tabular}

DE: desviación estándar.

tró un 42,5\% de casos de sexo masculino y una media de edad de 49,1 años. Así mismo, es similar a lo reportado por el Centro Nacional de Epidemiología, Prevención y Control de Enfermedades (CDCPerú), quienes mencionan que, en Perú, un 59,9\% de los casos se encuentran distribuidos en un rango de edad entre 30-59 años y un 41,8\% del sexo masculino 27.

El 38,4\% de los casos positivos refirieron tener alguna de las condiciones de riesgo, de acuerdo a normatividad vigente 28 . La condición de riesgo más frecuente fue la edad mayor o igual a 60 años (18,4\%); lo que es similar a lo encontrado por la CDC-Perú (17,3\% de casos con edad mayor o igual a 60 años) 27. Adicionalmente, Davies et al. 29 estiman que el 69\% de los casos en adultos mayores presenta síntomas clínicos, mientras que la susceptibilidad a la infección disminuye a la mitad en las personas menores de 20 años.

Otras condiciones de riesgo informadas en los casos secundarios fueron: hipertensión arterial $(7,2 \%)$, asma bronquial $(6,4 \%)$ y diabetes tipo 2 (5,6\%). Las investigaciones previas son contradictorias con respecto a la frecuencia de casos de hipertensión arterial encontrada en pacientes con COVID-19, siendo reportados desde el 1,9\% 30 hasta 17,4\% 26; mientras que, con respecto a la presencia de asma bronquial, se han encontrado frecuencias entre $8,8 \%-12,5 \% 31,32$, muy similares a la de esta investigación, y a lo reportado en Lima Metropolitana (18\%-19\%), por otras investigaciones 33,34. 
Por último, la frecuencia de diabetes tipo 2, encontrada en esta investigación es coherente con lo encontrado por Tabata et al. 30 , aunque es superior a lo reportado por otros estudios, en los que las frecuencias son, en promedio, un $3 \% 26,35$.

Por otro lado, la tríada de síntomas más frecuentemente encontrada fue la de fiebre, dolor de garganta y tos, observada en, aproximadamente, un 40\%-50\% de los casos sintomáticos positivos. Estos hallazgos son coherentes con estudios previos que evidencian que la fiebre y la tos eran los síntomas más frecuentes, presentes hasta en un $80 \%$ de los casos 26,36. Bi et al. 21 encontraron una relación estadísticamente significativa con una razón de prevalencia de 3,06 (IC95\%: 1,69-5,49) entre el hallazgo de fiebre y la detección de COVID-19. Si bien esta investigación no encontró una relación entre síntomas específicos y la positividad general de la prueba (IgG y/o IgM), lo estudiado sobre la historia natural de la enfermedad, indica que la aparición de IgM, previa a la de IgG, se da durante la primera-segunda semana después del inicio de síntomas ${ }^{37}$. No obstante, se debe considerar que, en el contexto actual, la aparición de aspectos semiológicos sugestivos, como los previamente mencionados, debe llevar a una sospecha diagnóstica racional, con la toma de decisión de aplicar una prueba para confirmarla.

Adicionalmente, el 22,4\% de los casos sintomáticos presentaron ageusia y el 20,8\%, anosmia. La evidencia aún no es clara con respecto a la frecuencia de estos hallazgos en casos de COVID-19, por un lado, algunas investigaciones estiman su presencia en más del 50\% de casos 38 ; sin embargo, esto no es respaldado por la evidencia nacional brindada por CDC-Perú, que reporta una frecuencia de $1,1 \%$ de anosmia y $0,3 \%$ de ageusia 27 . Sobre esto se debe considerar el notable sesgo de información que puede haber al respecto, ya que estos síntomas no eran frecuentemente consultados a los casos. Sin embargo, se evidencia que el 92,9\% y 90,3\% de los contactos con estos síntomas fueron positivos a la prueba serológica. Al respecto Patel et al. 38 reportaron que un $58 \%$ de contactos cohabitantes en pacientes con anosmia y COVID-19; también refirieron síntomas de esta enfermedad e incluso anosmia. Futuros estudios deberán evaluar mejor las características relacionadas a la aparición de estos síntomas y la transmisibilidad del SARS-CoV-2.

Esta investigación encontró un $22,4 \%$ de casos positivos, en calidad de asintomáticos, lo que es similar a la proporción de 29\% evidenciado por CDC-Perú 27. Así mismo, esto es coherente con lo encontrado en otros estudios de vigilancia de contactos, como el realizado por Bi et al. 21, en China, donde se encontró un $20 \%$ de casos secundarios asintomáticos; y por Cheng et al. 39, en Taiwán, quienes calcularon un $18,2 \%$ de casos secundarios en esta condición.

Por otro lado, al realizar la reevaluación de los casos se concluyó que, si bien la frecuencia de personas con síntomas bajó, la razón de casos positivos subió de 1,33 a 1,91. Esto es coherente con el tiempo de reevaluación (más de 30 días en promedio), debido a que la sensibilidad de la detección de anticuerpos en la población se incrementa de forma proporcional al tiempo de enfermedad 40. A pesar de ello, no se encontró un número importante de seroconversiones en las personas que salieron negativas en la primera evaluación.

Cabe destacar que en esta investigación también se ha caracterizado a los hogares como unidades de medida, estableciendo correcciones en los indicadores epidemiológicos por la densidad del hogar. Esta consideración es sumamente importante si se realiza un estudio de contactos en conglomerados específicos como las viviendas, sobre todo en un modo de selección no aleatorio, como en este caso. Es así que en el $23,1 \%$ de los hogares evaluados no se encontró ningún caso positivo, encontrándose una densidad promedio de 4,5 $\pm 2,5$ personas por hogar y de 3,7 $\pm 3,1$ personas, en los hogares donde todos los integrantes resultaron positivos. Esta densidad fue similar, y no explicaría la ausencia o mayor infección de contactos en estos hogares. Jing et al. 23 realizaron una experiencia similar, encontrando un $65 \%$ de hogares sin casos positivos, con una mediana de integrantes por hogar de $6(4,10)$ personas; sin embargo, no analizaron las características de los hogares con casos positivos.

Adicionalmente, y como era de esperarse, si se encuentra una diferencia importante en el porcentaje de personas con síntomas entre los hogares con todos los contactos positivos, respecto a los hogares con todos los contactos negativos (83,6\% vs. 48\%).

En relación a las diferencias entre hogares de distritos con baja carga de casos y hogares de distritos con alta carga, se evidencia que en los del primer grupo el porcentaje ponderado de contactos con signos y síntomas, y de contactos con resultados positivos es menor.

Esta investigación tiene importantes limitaciones que considerar; en primer lugar, la selección de las viviendas en la actividad de vigilancia epidemiológica se realizó por conveniencia, por lo que los 
resultados presentados no son extrapolables a la población en general. Así mismo, no hubo un componente de temporalidad, lo que no permite establecer si los casos denominados como "asintomáticos" fueron en realidad casos presintomáticos. Adicionalmente, la actividad de reevaluación no se pudo realizar en todos los hogares inicialmente incluidos, lo que agrega un importante sesgo de selección y disminuye la validez externa de las conclusiones que se puedan obtener de estos datos. A pesar de ello, debido a no encontrar antecedentes similares en Latinoamérica, este estudio presenta resultados que pueden servir como base para futuras investigaciones que generen conocimiento en torno a la dinámica de la transmisión en hogares del SARS-CoV-2.

A pesar de que las pruebas serológicas empleadas en el estudio cuentan con respaldo de la autoridad reguladora estatal (INS), a través de evaluaciones de laboratorio; en el Perú, solo existe un estudio de campo que emplea este tipo de pruebas para dicho fin; sin embargo, esta investigación utiliza otra marca (Zhejiang Orient Gene Biotech Co., Huzhou, China), y no define valores de rendimiento diagnóstico.

Finalmente, se concluye que al tener un caso primario de COVID-19 en el hogar, la tasa de ataque secundaria de esta infección es de 53\%; sin embargo, en un 23\% de los hogares evaluados no hubo un caso positivo, más allá del caso primario. Las características epidemiológicas y clínicas encontradas en este caso estuvieron acorde a lo ya reportado en otras series internacionales. De forma similar, la proporción de asintomáticos encontrada $(22,4 \%)$ es coherente con lo evidenciado por publicaciones previas y los datos epidemiológicos nacionales. Asimismo, se evidencia la persistencia de IgM positiva en la reevaluación de casos en promedio 30 días después.

\section{Colaboradores}

Todos los autores participarón de la concepción del proyecto, análisis e interpretación de los datos, redacción del artículo, revisión crítica relevante del contenido intelectual, aprobación final de la versión que debe ser publicada y, se hacen responsables de todos los aspectos del trabajo.

\section{Informaciones adicionales}

ORCID: Yolanda Angulo-Bazán (0000-0002-7280170X); Gilmer Solis-Sánchez (0000-0001-7084088X); Fany Cardenas (0000-0002-4541-275X); Ana Jorge (0000-0003-0570-4640); Joshi Acosta (0000-0001-5745-3383); César Cabezas (00000001-5120-0713).

\section{Agradecimientos}

A los Méd. Joel Roque Hernández y Duilio Fuentes Delgado por su colaboración técnica en la revisión y sugerencias durante el proceso de aprobación del protocolo de investigación. A el Instituto Nacional de Salud (Perú) por la financiación. A Lilyana Collazos y Lenin Rueda por el apoyo técnico brindado. 


\section{Referencias}

1. Rothan HA, Byrareddy SN. The epidemiology and pathogenesis of coronavirus disease (COVID-19) outbreak. J Autoimmun 2020; 109:102433.

2. Gobierno del Perú. Presidente Vizcarra dio a conocer primer caso de infección por coronavirus en el Perú e hizo un llamado a la población a mantener la calma. https://www.gob. pe/institucion/presidencia/noticias/86976presidente-vizcarra-dio-a-conocer-primercaso-de-infeccion-por-coronavirus-en-el-pe ru-e-hizo-un-llamado-a-la-poblacion-a-man tener-la-calma (accedido el 05/Abr/2020).

3. Ministerio de Salud. Minsa lamenta el sensible fallecimiento de dos personas por infección por COVID-19. https://www.gob.pe/institu cion/minsa/noticias/109603-minsa-lamentael-sensible-fallecimiento-de-dos-personaspor-infeccion-por-covid-19-comunicadon-21 (accedido el 05/Abr/2020).

4. Lake MA. What we know so far: COVID-19 current clinical knowledge and research. Clin Med (Lond) 2020; 20:124-7.

5. Liu Y, Gayle AA, Wilder-Smith A, Rocklöv J. The reproductive number of COVID-19 is higher compared to SARS coronavirus. J Travel Med 2020; $27:$ taaa021.

6. Riou J, Althaus CL. Pattern of early humanto-human transmission of Wuhan 2019 novel coronavirus (2019-nCoV), December 2019 to January 2020. Eurosurveillance 2020; 25:2000058.

7. Guo Y-R, Cao Q-D, Hong Z-S, Tan Y-Y, Chen $\mathrm{S}-\mathrm{D}$, Jin $\mathrm{H}-\mathrm{J}$, et al. The origin, transmission and clinical therapies on coronavirus disease 2019 (COVID-19) outbreak - an update on the status. Mil Med Res 2020; 7:11.

8. Wang Y, Wang Y, Chen Y, Qin Q. Unique epidemiological and clinical features of the emerging 2019 novel coronavirus pneumonia (COVID-19) implicate special control measures. J Med Virol 2020; 92:568-76.

9. Chih-Cheng L, Yen HL, Cheng-Yi W, Ya-Hui W, Shun-Chung H, Muh-Yen Y, et al. Asymptomatic carrier state, acute respiratory disease, and pneumonia due to severe acute respiratory syndrome coronavirus 2 (SARS-CoV-2): facts and myths. J Microbiol Immunol Infect 2020; 53:404-12.

10. Fan C, Liu L, Guo W, Yang A, Ye C, Jilili M, et al. Prediction of epidemic spread of the 2019 novel coronavirus driven by Spring Festival transportation in China: a population-based study. Int J Environ Res Public Health 2020; 17:1679.

11. Liu Y, Eggo RM, Kucharski AJ. Secondary attack rate and superspreading events for SARSCoV-2. Lancet 2020; 395:e47.

12. Burke RM, Midgley CM, Dratch A, Fenstersheib M, Haupt T, Holshue M, et al. Active monitoring of persons exposed to patients with confirmed COVID-19 - United States, January-February 2020. MMWR Morb Mortal Wkly Rep 2020; 69:245-6.
13. Wu JT, Leung K, Bushman M, Kishore N, Niehus R, Salazar PM, et al. Estimating clinical severity of COVID-19 from the transmission dynamics in Wuhan, China. Nat Med 2020; 26:506-10.

14. Burki T. COVID-19 in Latin America. Lancet Infect Dis 2020; 20:547-8.

15. Benítez MA, Velasco C, Sequeira AR, Henríquez J, Menezes FM, Paolucci F. Responses to COVID-19 in five Latin American countries. Health Policy Technol 2020; 9:525-59.

16. Moller S. Intervenciones sociosanitarias y uso de las tecnologías de la industria 4.0 para enfrentar la enfermedad por coronavirus (COVID-19) en América Latina y el Caribe. Santiago: Comisión Económica para América Latina y el Caribe; 2020.

17. Munayco CV, Tariq A, Rothenberg R, SotoCabezas GG, Reyes MF, Valle A, et al. Early transmission dynamics of COVID-19 in a southern hemisphere setting: Lima-Peru, February 29th-March 30th, 2020. Infect Dis Model 2020; 5:338-45.

18. Centro Nacional de Salud Pública, Instituto Nacional de Salud. Nota Informativa $n^{\circ}$ 072-2020-EQ-PR-COVID19. Lima: Instituto Nacional de Salud; 2020.

19. Ministerio de Salud. Resolución Ministerial no 193-2020, de 13 de abril de 2020. Prevención, diagnóstico y tratamiento de personas afectadas por COVID-19 en el Perú. Lima: Ministério de Salud; 2020.

20. Wu J, Huang Y, Tu C, Bi C, Chen Z, Luo L, et al. Household transmission of SARS-CoV-2, Zhuhai, China, 2020. Clin Infect Dis 2020; 71:2099-108.

21. Bi Q, Wu Y, Mei S, Ye C, Zou X, Zhang Z, et al. Epidemiology and transmission of COVID-19 in 391 cases and 1286 of their close contacts in Shenzhen, China: a retrospective cohort study. Lancet Infect Dis 2020; 20:911-9.

22. COVID-19 National Emergency Response Center, Epidemiology and Case Management Team; Korea Centers for Disease Control and Prevention. Coronavirus disease-19: summary of 2,370 contact investigations of the first 30 cases in the Republic of Korea. Osong Public Health Res Perspect 2020; 11:81-4.

23. Jing Q-L, Liu M-J, Zhang Z-B, Fang L-Q, Yuan J, Zhang A-R, et al. Household secondary attack rate of COVID-19 and associated determinants in Guangzhou, China: a retrospective cohort study. Lancet Infect Dis 2020; 20:114150.

24. Guan Q, Liu M, Zhuang YJ, Yuan Y, Wang SS, $\mathrm{Li} \mathrm{J}$, et al. Epidemiological investigation of a family clustering of COVID-19. Zhonghua Liu Xing Bing Xue Za Zhi 2020; 41:629-33.

25. Qian G, Yang N, Ma AHY, Wang L, Li G, Chen $\mathrm{X}$, et al. COVID-19 transmission within a family cluster by presymptomatic carriers in China. Clin Infect Dis 2020; 71:861-2. 
26. Lovato A, De Filippis C. Clinical presentation of COVID-19: a systematic review focusing on upper airway symptoms. Ear Nose Throat J 2020; 99:569-76.

27. Centro Nacional de Epidemiología, Prevención y Control de Enfermedades. Situación actual "COVID-19" al 21 de junio 2020. Lima: Centro Nacional de Epidemiología, Prevención y Control de Enfermedades; 2020.

28. Ministerio de Salud. Resolución Ministerial no 240-2020, de 29 de abril de 2020. Modifican el Documento Técnico: Prevención Diagnóstico y Tratamiento de personas afectadas por COVID-19 en el Perú aprobado por R.M. № 193-2020-MINSA. Lima: Ministério de Salud; 2020.

29. Davies NG, Klepac P, Liu Y, Prem K, Jit M, Eggo RM. Age-dependent effects in the transmission and control of COVID-19 epidemics. Nature Med 2020; 26:1205-11.

30. Tabata S, Imai K, Kawano S, Ikeda M, Kodama $\mathrm{T}$, Miyoshi K, et al. Clinical characteristics of COVID-19 in 104 people with SARS-CoV-2 infection on the Diamond Princess cruise ship: a retrospective analysis. Lancet Infect Dis 2020; 20:1043-50.

31. Butler MW, O'Reilly A, Dunican EM, Mallon P, Feeney ER, Keane MP, et al. Prevalence of comorbid asthma in COVID-19 patients. J Allergy Clin Immunol 2020; 146:334-5.

32. Goyal P, Choi JJ, Pinheiro LC, Schenck EJ, Chen R, Jabri A, et al. Clinical characteristics of COVID-19 in New York City. New Engl J Med 2020; 382:2372-4.

33. Robinson CL, Baumann LM, Gilman RH, Romero K, Combe JM, Cabrera L, et al. The Peru urban versus rural asthma (PURA) Study: methods and baseline quality control data from a cross-sectional investigation into the prevalence, severity, genetics, immunology and environmental factors affecting asthma in adolescence in Peru. BMJ Open 2012; 2:e000421.
34. Lai CKW, Beasley R, Crane J, Foliaki S, Shah J, Weiland S, et al. Global variation in the prevalence and severity of asthma symptoms: phase three of the International Study of Asthma and Allergies in Childhood (ISAAC). Thorax 2009; 64:476-83.

35. Nikpouraghdam M, Jalali Farahani A, Alishiri G, Heydari S, Ebrahimnia M, Samadinia H, et al. Epidemiological characteristics of coronavirus disease 2019 (COVID-19) patients in IRAN: a single center study. J Clin Virol 2020; 127:104378

36. Fu L, Wang B, Yuan T, Chen X, Ao Y, Fitzpatrick $\mathrm{T}$, et al. Clinical characteristics of coronavirus disease 2019 (COVID-19) in China: a systematic review and meta-analysis. J Infect 2020; 80:656-65.

37. Sethuraman N, Jeremiah SS, Ryo A. Interpreting diagnostic tests for SARS-CoV-2. JAMA 2020; 323:2249-51.

38. Patel A, Charani E, Ariyanayagam D, Abdulaal A, Denny SJ, Mughal N, et al. New-onset anosmia and ageusia in adult patients diagnosed with SARS-CoV-2 infection. Clin Microbiol Infect 2020; 26:1236-41.

39. Cheng H-Y, Jian S-W, Liu D-P, Ng T-C, Huang W-T, Lin $\mathrm{H}-\mathrm{H}$. Contact tracing assessment of COVID-19 transmission dynamics in taiwan and risk at different exposure periods before and after symptom onset. JAMA Intern Med 2020; 80:1156-63.

40. Zhao J, Yuan Q, Wang H, Liu W, Liao X, Su $\mathrm{Y}$, et al. Antibody responses to SARS-CoV-2 in patients of novel coronavirus disease 2019. Clin Infect Dis 2020; ciaa344. 
Abstract

The study aimed to describe the characteristics of SARS-CoV-2 transmission among members of households with a confirmed primary case of COVID-19 in districts with low burden of cases in Lima, Peru, compared to a district with high burden. This was a retrospective study with a secondary database review. Information was collected from an epidemiological surveillance activity in close contacts (household members) in 52 households in Lima, with a single member with COVID-19. Reevaluation was conducted in 10 households. The study evaluated epidemiological and clinical variables and their association with the result of the rapid serological test (presence of $\operatorname{IgG}, \operatorname{Ig} M$, or both). Secondary cases were found in 40 households, representing mean identification of $49.9 \%$ per household. Secondary attack rate in household members was 53\% (125 cases), and symptomatic individuals accounted for $77.6 \%$ of cases (symptomatic/asymptomatic ratio: 3.5 ). Presence of fever and/or chills was found in 40\% of persons with positive test results, followed by sore throat with $39.2 \%$. Ageusia and anosmia were present in $22.4 \%$ and $20.8 \%$ of cases, respectively. When there was a primary case of COVID-19 in the household, the secondary attack rate was 53\%; however, in an important proportion of households there were no positive cases other than the primary case. The epidemiological and clinical findings were consistent with reports from other international series.

COVID-19; Contact Tracing; Epidemiologic Surveillance Services

\section{Resumo}

O objetivo foi descrever as características da infecção por SARS-CoV-2 entre os membros de domicílios, com um caso primário confirmado de COVID-19, em distritos com baixa carga de casos em Lima, Peru, em comparação com um distrito com alta carga. Estudo retrospectivo de revisão de banco de dados secundário. As informações foram coletadas em uma atividade de vigilância epidemiológica em contatos próximos (coabitantes), em 52 domicílios em Lima, com um único membro com COVID-19. Foi realizada uma reavaliação em 10 domicílios. Variáveis epidemiológicas e clínicas foram avaliadas; e sua associação com o resultado do teste sorológico rápido (presença de IgG, IgM ou ambos). Os casos secundários foram encontrados em 40 domicílios, representando uma taxa média de identificação de 49,9\% por domicílio. Foi encontrada uma taxa de ataque secundário entre coabitantes de 53\% (125 casos), com 77,6\% dos casos sendo sintomáticos (relação sintomático/assintomático: 3,5). A presença de febre e/ou calafrios foi encontrada em $40 \%$ das pessoas com resultado positivo, seguida de dor de garganta em $39,2 \%$. Ageusia e anosmia estiveram presentes em $22,4 \%$ e 20,8\% dos casos, respectivamente. Quando há um caso primário de COVID-19 em casa, a taxa de ataque secundário para essa infecção é de 53\%; No entanto, em uma proporção significativa dos domicílios avaliados não houve caso positivo, além do caso primário. As características epidemiológicas e clínicas encontradas neste caso foram consistentes com o que já foi relatado em outras séries internacionais.

COVID-19; Busca de Comunicante; Serviços de Vigilância Epidemiológica
Recibido el 11/Ago/2020

Versión final presentada el 19/Oct/2020

Aprobado el 27/Nov/2020 